\title{
Effects of high-intensity interval versus continuous moderate-intensity aerobic exercise on apoptosis, oxidative stress and metabolism of the infarcted myocardium in a rat model
}

\author{
KAI LU ${ }^{1,2}$, LI WANG ${ }^{2}$, CHANGYING WANG ${ }^{2}$, YUAN YANG ${ }^{2}$, DAYI HU ${ }^{1}$ and RONGJING DING ${ }^{1}$ \\ ${ }^{1}$ Heart Center, Peking University People's Hospital, Beijing 100044; ${ }^{2}$ Department of Cardiology, \\ The First Affiliated Hospital of Chongqing Medical University, Chongqing 400016, P.R. China
}

Received June 30, 2014; Accepted March 10, 2015

DOI: $10.3892 / \mathrm{mmr} .2015 .3669$

\begin{abstract}
The optimal aerobic exercise training (AET) protocol for patients following myocardial infarction (MI) has remained under debate. The present study therefore aimed to compare the effects of continuous moderate-intensity training (CMT) and high-intensity interval training (HIT) on cardiac functional recovery, and to investigate the potential associated mechanisms in a post-MI rat model. Female Sprague Dawley rats (8-10 weeks old) undergoing MI or sham surgery were subsequently submitted to CMT or HIT, or kept sedentary for eight weeks. Prior to and following AET, echocardiographic parameters and exercise capacity of the rats were measured. Western blotting was used to evaluate the levels of apoptosis and associated signaling pathway protein expression. The concentrations of biomarkers of oxidative stress were also determined by ELISA assay. Messenger (m)RNA levels and activity of the key enzymes for glycolysis and fatty acid oxidation, as well as the rate of adenosine triphosphate (ATP) synthesis, were also measured. Compared with the MI group, exercise capacity and cardiac function were significantly improved following AET, particularly following HIT. Left ventricular ejection fraction and fraction shortening were further improved in the MI-HIT group in comparison to that of the MI-CMT group. The two forms of AET almost equally attenuated apoptosis of the post-infarction myocardium. CMT and HIT also alleviated oxidative stress by decreasing the concentration of malondialdehyde and increasing the concentration of superoxide dismutase and glutathione peroxidase (GPx). In particular, HIT induced a greater increase in the concentration of GPX than that of CMT. AET, and HIT in particular, significantly increased the levels of mRNA and the maximal activity of phosphofructokinase-1 and carnitine palmitoyl transferase-1,
\end{abstract}

Correspondence to: Dr Rongjing Ding, Heart Center, Peking University People's Hospital, 11 South Xizhimen Avenue, Beijing 100044, P.R. China E-mail: rongjingding@163.com

Key words: aerobic exercise training, myocardial infarction, cardiac function as well as the maximal ratio of ATP synthesis. In addition, compared with the MI group, the expression of signaling proteins PI3K, Akt, p38mapk and AMPK was significantly altered in the MI-CMT and MI-HIT groups. HIT was superior to CMT in its ability to improve cardiac function and exercise capability in a post-MI rat model. HIT was also superior to CMT with regard to attenuating oxidative stress and improving glucolipid metabolism of the post-MI myocardium.

\section{Introduction}

The benefits of aerobic exercise training (AET) for patients with myocardial infarction (MI) are well-documented in the literature (1-3). Continuous moderate training (CMT) and high-intensity interval training (HIT) are the two forms of AET, which have been most studied in previous research, and a growing body of evidence has demonstrated that HIT is superior to CMT in terms of enhancing exercise capability (4-6). Cardiac functional recovery is another focus in the process of cardiac rehabilitation for patients with MI (7). However, the effects of CMT and HIT on cardiac functional recovery have not been well documented. Skeletal muscle and cardiopulmonary adaption to aerobic training are the major reasons for the improvement in exercise capacity in MI patients following cardiac rehabilitation, although other elements may also contribute $(8,9)$. Moreira et al (10) reported that skeletal muscle adaption to exercise training was notably similar between CMT and HIT. Based on the above, it was suggested that a comparison of the benefits of CMT and HIT on cardiac functional recovery may provide evidence for further exploration of the precise mechanisms of metabolism underlying the superior outcomes produced by HIT.

In addition, apoptosis and oxidative stress are involved in the development of multiple cardiovascular diseases, including MI, and mounting evidence has shown that alleviation of oxidative injury and myocardial apoptosis may exert cardioprotective effects and improve recovery of cardiac function $(11,12)$. Although previous reports have documented that AET may attenuate cardiac apoptosis and oxidative stress in the post-MI heart $(13,14)$, the effects of distinct exercise protocols on these factors have rarely been directly compared. 
Altering energy metabolism by decreasing fatty acid oxidation or increasing glucose oxidation may improve cardiac recovery following an ischemic insult $(15,16)$. Abnormal glucose metabolism was also found to be associated with reduced left ventricular contractile reserve and exercise intolerance in patients with chronic heart failure (17). It has been confirmed that AET is able to decrease the insulin sensitivity and increase the glucose uptake of the skeletal muscles of animals and humans (18-20). Furthermore, it was reported that exercise training is able to effectively prevent the depression in myocardial glucose metabolism observed in the diabetic rat (20). Based on these results, it was hypothesized that aerobic exercise may influence glucose and fatty acid metabolism in the infarcted myocardium, and that this may contribute to the recovery of cardiac function induced by AET.

In the present study, the effects of two AET protocols (HIT and CMT) on cardiac functional recovery and exercise capability were compared. Furthermore, the impact of HIT and CMT on potentially associated mechanisms, including apoptosis, oxidative stress and glucolipid metabolism, were investigated. The phosphatidylinositol-3-kinase/serine/threonine kinase (PI3K/Akt), p38 mitogen-activated protein kinase (p38mapk) and adenosine monophosphate activated protein kinase (AMPK) signaling pathways are widely agreed to be pivotal signal pathways, which have key regulatory roles in cellular apoptosis (21), oxidative stress (22) and fatty acid metabolism (23), respectively. Alterations in their expression levels following various AET protocols were also evaluated in the present study.

\section{Materials and methods}

Animal models. Female Sprague Dawley rats $(n=40)$ aged 8-10 weeks were provided by the Animal Center of China Academy of Military Medical Science (Beijing, China) and were randomly divided into groups (10/group). The rats were maintained at $20-24^{\circ} \mathrm{C}$ with $40-60 \%$ humidity under a $12 / 12 \mathrm{~h}$ light/dark cycle, receiving standard chow and water ad libitum. Studies commenced following one week of rat acclimatization. MI was induced as previously described by Pfeffer et al (24). The rats were fully anaesthetized by intraperitoneal injection of $10 \mathrm{ml} / \mathrm{kg} \mathrm{4 \%}$ amobarbitalum (AstraZeneca, Shanghai, China) and artificially ventilated. The heart was exposed by a left thoracotomy between the fourth and fifth ribs. For the animals in which MI was induced, a 6-0 mononylon suture was passed under the main left descending coronary artery at the point between 1 and $2 \mathrm{~mm}$ distal to the edge of the left atrium, and the left coronary artery was ligated. Age-matched rats in the sham group underwent the same procedure without ligation of left descending coronary artery. Subsequently the thorax was closed, the skin was sutured and the pneumothorax was drained by a continuous aspiration system. The study was conducted according to the Guide for the Care and Use for Laboratory Animals (version 1; 1983; National Institutes of Health, Beijing, China) and approved by the Ethics Committee of Peking University People's Hospital (Beijing, China).

Aerobic exercise protocols. AET was performed on a WI-78059 treadmill specifically designed for small animals
(Yiyan Technology, Jinan, China). Animals were adapted to treadmill exercise for two weeks, gradually increasing from 10 to $30 \mathrm{~min} /$ day. An eight-week exercise protocol began, following this adaptation period. The rats in the exercise groups were subjected to either CMT or HIT five days per week. CMT was performed at a constant running speed corresponding to $50-60 \%$ of maximal oxygen uptake $\left(\mathrm{VO}_{2} \mathrm{max}\right)$ throughout the training session (50 min/day). HIT included training sessions which comprised 4 min running at $85-90 \% \mathrm{VO}_{2} \max$ followed by $3 \mathrm{~min}$ running at $50-60 \%$ $V \mathrm{O}_{2}$ max, repeated seven times (49 min training in total). Prior to and following the exercise period, there was a $5 \mathrm{~min}$ warm-up and cool-down at $40 \% \mathrm{VO}_{2}$ max. Rats in the sham and MI groups were not subjected to any additional exercise. Running intensity for each protocol was based on a previous report outlining the association between running speed and $\mathrm{VO}_{2}$ max in an identical rat model of $\mathrm{MI}(25,26)$.

Echocardiography. Echocardiographic evaluation was performed prior to and following the exercise training, using the Vevo770 ultrasound system (Visualsonics Inc., Toronto, ON, Canada). Animals were anesthetized by intraperitoneal injection of $4 \%$ amobarbitalum $(10 \mathrm{ml} / \mathrm{kg})$ and placed in a supine position on the examination table. The probe frequency was set at $17.5 \mathrm{MHz}$, sampling frequency was in M-mode at 1000/s and the scanning speed was $50-100 \mathrm{~mm} / \mathrm{s}$. The probes were placed on the precordium and the detection was conducted from the section of the ventricular bands. The left ventricular end-diastolic diameters (LVEDD) and left ventricular end-systolic diameters (LVESD) were measured and the left ventricular end-diastolic volume (LVEDV), left ventricular end-systolic volume (LVESV), left ventricular ejection fraction (LVEF) and left ventricular fraction shortening (LVFS) were calculated based on these measurements, using the following formulae: $\mathrm{LVEDV}=\left(7.0 \times \mathrm{LVEDD}^{3}\right) /(2.4+\mathrm{LVEDD})$; $\mathrm{LVESV}=\left(7.0 \times \mathrm{LVESD}^{3}\right) /(2.4+\mathrm{LVESD})$; LVEF=(LVEDV-LVESV)/LVEDVx $100 \%$; LVFS $=($ LVEDD-LVESD)/LVEDDx $100 \%$.

Exercise capability and aerobic capacity measurement. Exercise capability was measured prior to and following the exercise training. The method was similar to that previously described by Moreira et al (10). Briefly, the rats ran on a graded treadmill at $15^{\circ}$ inclination at an initial speed of $6 \mathrm{~m} / \mathrm{min}$. The speed was subsequently increased by $3 \mathrm{~m} / \mathrm{min}$ every $3 \mathrm{~min}$ until the rats were unable to run. The total distance run by each rat was considered to indicate the exercise capability.

Tissue preparation. Rats were sacrificed by decapitation once the eight-week exercise protocol ended, and the hearts were harvested as quickly as possible. The atriums and right ventricles were trimmed off and the left ventricles were stored in liquid nitrogen (Chaoyang Gas Plant, Beijing, China) for future evaluation. For measurement of the activity of carnitine palmitoyl transferase-1 (CPT-1) and the rate of adenosine triphosphate (ATP) synthesis, fresh heart tissues were used and the experiments were completed within $4 \mathrm{~h}$ of the harvest of the heart. 
Table I. Primer sequences for CPT-1, PFK-1 and GAPDH.

\begin{tabular}{ll}
\hline Gene name & \multicolumn{1}{c}{ Primer sequence } \\
\hline CPT-1 F & 5'-AAGAACACGAGCCAACAAGC-3' \\
CPT-1 R & 5'-TACCATACCCAGTGCCATCA-3' \\
PFK-1 F & 5'-GATGCCCAAGGTATGAATGC-3' \\
PFK-1 R & 5'-CTCCCTGATGTGCTCTCCAC-3' \\
GAPDH F & 5'-CCCTTCATTGACCTCAACTACATG-3' \\
GAPDH R & 5'-CTTCTCCATGGTGGTGAAGAC-3' \\
\hline
\end{tabular}

CPT-1, carnitine palmitoyltransferase-1; PKF-1, phosphofructokinase $1 ; \mathrm{F}$, forward; $\mathrm{R}$, reverse.

Measurement of biomarkers of oxidative stress. The concentrations of malondialdehyde (MDA), glutathione peroxidase (GPx) and superoxide dismutase (SOD) in the heart homogenate were determined by ELISA assay. The experiment was performed using a commercially available kit (ELISA kit for the Measurement of Oxidative Stress in Rat) according to the manufacturer's instructions (Jiancheng Bioengineering Institute, Nanjing, China). Briefly, heart tissues were collected and lysed with cell lysis buffer (in ELISA kit). Then cell lysates were centrifuged at $1600 \mathrm{x}$ g for $10 \mathrm{~min}$ at $4^{\circ} \mathrm{C}$ and the supernatants were collected for the detection of MDA, GPx and SOD. Following incubation with the reagents included in the respective kits, the absorbance values at $450 \mathrm{~nm}, 412 \mathrm{~nm}$ and $532 \mathrm{~nm}$ were measured using a spectrophotometer (721D; Pudong Shanghai Physical Optical Instrument Factory, Shanghai, China).

Reverse transcription-quantitative polymerase chain reaction (RT-qPCR). The messenger (m)RNA levels of CPT-1 and phosphofructokinase (PKF-1) were evaluated by RT-qPCR. Cardiac tissues were homogenized in TRIzol agent (Invitrogen Life Technologies, Carlsbad, CA, USA) and total RNA was extracted and reverse-transcribed into cDNA using the PrimeScript RT reagent kit (Takara Biotechnology, Co., Ltd., Dalian, China) with an Oligo dT primer. All samples were run in triplicate in a total reaction volume of $25 \mu 1$, which was comprised of $12.5 \mu \mathrm{l}$ SYBR Premix Ex TaqII, $2 \mu$ l primer, $2 \mu \mathrm{l}$ templates ( $1 \mu \mathrm{g}$ cDNA) and $8.5 \mu \mathrm{l}$ nuclease-free water. RT-qPCR was performed using the SYBR Premix Ex TaqII (Takara Biotechnology, Co., Ltd.) and two-step Real Time PCR system (Thermal Cycle Dice; Takara Bio, Inc., Otsu, Japan) in triplicate wells. The cycling parameters were as follows: Activation at $95^{\circ} \mathrm{C}$ for $30 \mathrm{sec}, 40$ cycles of denaturation at $95^{\circ} \mathrm{C}$ for $5 \mathrm{sec}$, and then annealing and extension at $60^{\circ} \mathrm{C}$ for $30 \mathrm{sec}$. The rat GAPDH gene was used as a reference. The primers used in the present study are presented in Table I. The normalized fold-changes of the target gene mRNA expression were expressed as $2^{-\Delta \Delta C t}$.

Measurement of CPT-1 and PFK-1 activity. At the time of sacrifice, mitochondria were isolated from the myocardium by differential centrifugation. Briefly, $200 \mathrm{mg}$ fresh ventricle tissue was homogenized with ice-cold lysis buffer and the homogenate was centrifuged at $800 \mathrm{xg}$ for $5 \mathrm{~min}$ at $4^{\circ} \mathrm{C}$. The supernate was collected and added to another tube containing medium buffer and then centrifuged at $15,000 \mathrm{x}$ g for $10 \mathrm{~min}$ at $4^{\circ} \mathrm{C}$. The sediment was kept, resuspended in wash buffer and centrifuged at $15,000 \mathrm{xg}$ for $10 \mathrm{~min}$ at $4^{\circ} \mathrm{C}$. The supernate was removed and finally the mitochondria of the ventricle tissue were obtained. The kit used in this protocol was provided by Beyotime Institute of Biotechnology (Haimen, China).

The CPT-1 activity of the myocardium was measured as previously reported (27). Briefly, assays were performed in $1 \mathrm{ml}$ reaction medium containing $220 \mathrm{mM}$ sucrose, $40 \mathrm{mM}$ $\mathrm{KCl}, 10 \mathrm{mM}$ Tris and $1 \mathrm{mM}$ ethylene glycol tetraacetic acid (EGTA), as well as mitochondrial inhibitors rotenone $(1 \mathrm{pg} / \mathrm{ml})$, antimycin A $(0.5 \mathrm{pg} / \mathrm{ml})$ and oligomycin $(1 \mathrm{pg} / \mathrm{ml})$ at $\mathrm{pH} 7.0$, at $30^{\circ} \mathrm{C}$. Reactions were initiated by the addition of $0.60 \mathrm{mg}$ mitochondrial protein and were stopped with $2 \mathrm{ml}$ butanol-saturated $0.73 \mathrm{M} \mathrm{HCl} 10 \mathrm{~min}$ later. The product was extracted into butanol $(1 \mathrm{ml})$ and following centrifugation $\left(10,000 \mathrm{x} \mathrm{g} ; 10 \mathrm{~min} ; 4^{\circ} \mathrm{C}\right.$ and washing with $2 \mathrm{ml}$ butanol-saturated $0.5 \mathrm{M}$ sodium phosphate $(\mathrm{pH} 7.0), 0.5 \mathrm{ml}$ of the butanol layer was used for final determination. The reaction product was measured every 5 min until the palmitoyl-CoA concentration was low (10-20 $\mu \mathrm{M})$, at which point the reaction product was measured every $2 \mathrm{~min}$. All the chemicals used in this protocol were provided by Sigma-Aldrich Shanghai Trading Co. Ltd (Shanghai, China).

The PFK-1 activity of the myocardium was also measured. In brief, assays were conducted in reaction medium containing $50 \mathrm{~mol} / 1 \mathrm{Hepes}$ buffer, $10 \mathrm{~mol} / \mathrm{l} \mathrm{HCl}, 6.5 \mathrm{~mol} / 1 \mathrm{MgCl}_{2}, 1 \mathrm{~mol} / 1$ $\mathrm{NH}_{4} \mathrm{Cl}, 5 \mathrm{~mol} / 1 \mathrm{KH}_{2} \mathrm{PO}_{4}, 0.1 \mathrm{~mol} / 1$ adenosine monophosphate, $0.3 \mathrm{~mol} / 1 \mathrm{NADH}, 0.5 \mathrm{U} / \mathrm{ml}$ aldolase, $0.5 \mathrm{U} / \mathrm{ml}$ glutamate dehydrogenase, $5 \mathrm{U} / \mathrm{ml}, 0.1 \mathrm{~mol} / 1$ fructose 6-phosphate, $0.3 \mathrm{~mol} / 1$ glucose 6-phosphate and 1.5-mol/1 ATP (pH 7.0). The activity of PFK-1 was assayed by monitoring the oxidation of NADH at $340 \mathrm{~nm}$ using a spectrophotometer (721D; Pudong Shanghai Physical Optical Instrument Factory) consecutively when triosephosphate isomerase and glycerophosphate were added into the reaction medium. All the chemicals used in this protocol were provided by Sigma-Aldrich Shanghai Trading Co. Ltd.

Measurement of the rate of ATP synthesis. The rate of ATP synthesis was modulated by glucose 6-phosphate accumulation using an adenosine diphosphate (ADP)-regenerating system based on hexokinase plus glucose and ATP which was described previously (28). The reaction medium $(120 \mathrm{mM}$ $\mathrm{KCl}, 5 \mathrm{mM} \mathrm{K \textrm {H } _ { 2 } \mathrm { PO } _ { 4 } , 1 \mathrm { mM } \text { EGTA, } 2 \mathrm { mM } \mathrm { MgCl }}$, $3 \mathrm{mM}$ Hepes; $\mathrm{pH}$ 7.4) was supplemented with $5 \mathrm{mM}$ succinate, $5 \mu \mathrm{M}$ rotenone, $20 \mathrm{mM}$ glucose and $125 \mu \mathrm{M}$ ATP to initiate the reaction. Glucose 6-phosphate formation was monitored at $37^{\circ} \mathrm{C}$ from NADPH content by spectrophotometry at $340 \mathrm{~nm}$ (721D; Pudong Shanghai Physical Optical Instrument Factory). All the chemicals used in this protocol were provided by Sigma-Aldrich Shanghai Trading Co. Ltd.

Western blot. Total protein was exacted from left ventricle myocardium lysates following centrifugation at 14,000 $\mathrm{x} g$ for $10 \mathrm{~min}$ at $4^{\circ} \mathrm{C}$. Proteins were separated by $12 \%$ SDS-PAGE and transferred onto a nitrocellulose membrane (Beyotime Institute of Biotechnology). The membrane was then blocked with 5\% non-fat dry milk and incubated in solution containing primary antibodies overnight. The following primary antibodies were 


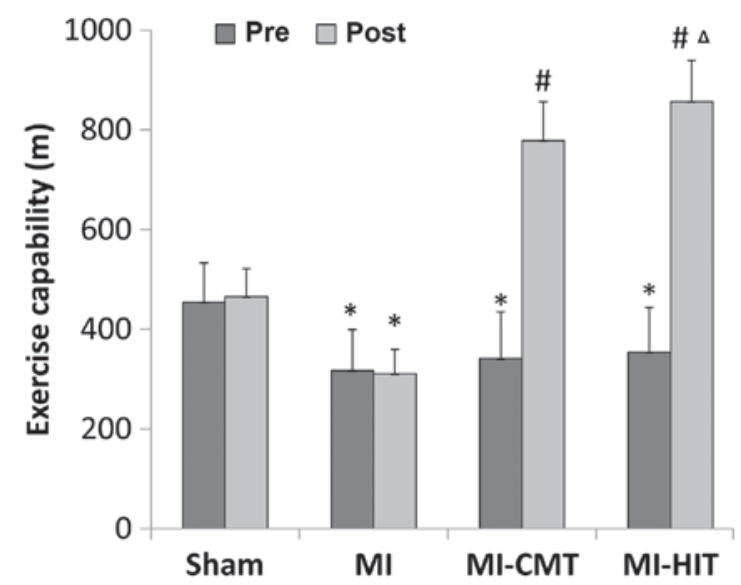

Figure 1. Effects of CMT and HIT on the exercise capacity of post-MI rats. Exercise capacity was measured by distance (m) that rats ran on a gradually accelerating treadmill until exhaustion. Data are expressed as the mean \pm standard deviation. ${ }^{*} \mathrm{P}<0.05$ vs. the sham group; ${ }^{*} \mathrm{P}<0.05$ vs. the MI group; ${ }^{\wedge} \mathrm{P}<0.05$ vs. the MI+CMT group. Sham, sham group; MI, rats rested following $\mathrm{MI}$; MI+CMT, rats subjected to continuous moderate intensity training post-MI; MI+HIT, rats subjected to high-intensity interval training post-MI. MI, myocardial infarction; CMT, continuous moderate training; HIT, high-intensity interval training.

used in the present study: Anti-bax antibody (rabbit/rat; polyclonal; 1:1,000; \#2772; Cell Signaling Technology, Inc., Danvers, MA, USA), anti-bcl-2 antibody (rabbit/rat; polyclonal IgG; 1:100; sc-492; Santa Cruz Biotechnology, Inc., Dallas, TX, USA), anti-PI3K antibody (rabbit/rat; polyclonal IgG; 1:100; sc-67306; Santa Cruz Biotechnology, Inc.), anti-phosphorylated-AKT $\mathrm{T}^{\mathrm{ser} 73}$ antibody (rabbit/rat; polyclonal IgG; 1:100; sc-33437; Santa Cruz Biotechnology, Inc.), anti-p38mapk antibody (rabbit/rat; polyclonal; 1:1,000; sc-728; Santa Cruz Biotechnology, Inc.), anti-phosphorylated-AMPK ${ }^{\text {Thr172 }}$ antibody (rabbit/rat; monoclonal; 1:1,000; \#5759; Cell Signaling Technology, Inc.) and anti- $\beta$-actin antibody (rabbit/rat; polyclonal; 1:1,000; sc-130657; Santa Cruz Biotechnology, Inc.). The membrane was subsequently incubated with the corresponding secondary antibody (goat/rabbit; polyclonal; 1:1,000; A0208; Beyotime Institute of Biotechnology) for $30 \mathrm{~min}$ the next day. Blots were imaged using the Bio-Rad gel imaging system (Bio-Rad Laboratories, Inc., Hercules, CA, USA) and the densities of bands were quantified using Quantity One software, version 4.5 (Bio-Rad Laboratories, Inc.).

Statistical analysis. All results are presented as the mean \pm standard deviation. Differences among grouped data were analyzed using a one-way analysis of variance followed by Dunnett's post-hoc test or the Mann-Whitney Rank sum test. $\mathrm{P}<0.05$ was considered to indicate a statistically significant difference. All data was analyzed with SPSS 19.0 software (IBM SPSS, Armonk, NY, USA).

\section{Results}

HIT is superior to CMT with regard to enhancing exercise capability. As indicated in Fig. 1, the baseline exercise capability of the rats in the MI-treated groups (MI, MI+CMT and MI+HIT) was significantly inferior to that of the sham group $(\mathrm{P}<0.05)$ and no significant differences were observed among 

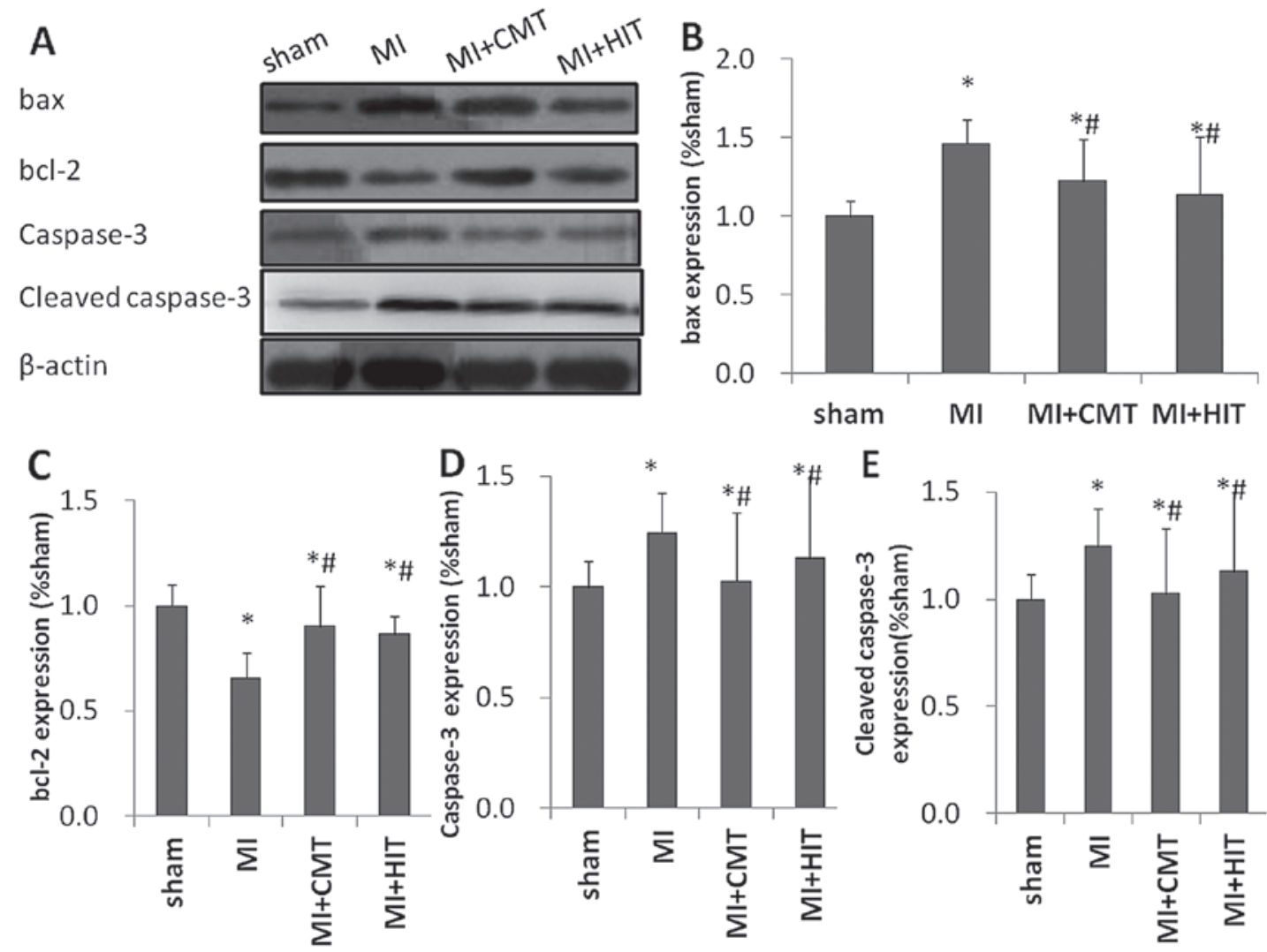

Figure 2. Effects of CMT and HIT on the expression of anti-apoptotic and pro-apoptotic proteins in the post-MI myocardium. (A) Representative western blot analysis results. Effects of CMT and HIT on protein expression of (B) bax, (C) bcl-2, (D) caspase-3 and (E) cleaved caspase-3. Protein expression levels were measured by western blotting. The data were normalized to the sham group and expressed as the mean \pm standard deviation of three independent experiments per group. ${ }^{*} \mathrm{P}<0.05$ vs. the sham group; ${ }^{\#} \mathrm{P}<0.05$ vs. the MI group. MI, myocardial infarction; CMT, continuous moderate training; HIT, high-intensity interval training; bcl-2, B cell lymphoma-2; bax, bcl-2-associated protein $\mathrm{X}$.

the various MI-treated groups. Following the training period, CMT markedly enhanced the exercise capability of rats compared with that of the MI group, in which rats remained sedentary for the majority of the time. However, HIT was able to improve exercise capability to a greater extent and rats ran $\sim 100 \mathrm{~m}$ more than those in the CMT group (856.5 \pm 82.6 vs. 778.12 $\pm 78.1 ; \mathrm{P}<0.05)$.

HIT improves post-MI cardiac function more than CMT. LVEF and FS in the MI-treated groups were significantly decreased compared with those of the sham group, and no significant differences were observed among the various MI-treated groups prior to exercise training (Table II). When exercise training ended, LVEF and FS in the two exercise training groups were significantly increased compared with those of the MI group. In particular, LVEF and FS were better restored in the HIT group than the CMT group (change of LVEF: $20.4 \pm 0.52 \%$ vs. $10.4 \pm 0.39 \%, \mathrm{P}<0.05$; change of FS: $5.7 \pm 0.70 \%$ vs. $10.3 \pm 0.55 \%, \mathrm{P}<0.05)$.

CMT and HIT attenuate apoptosis of the infarcted myocardium. As shown in Fig. 2, expression levels of pro-apoptotic proteins B cell lymphoma 2 (bcl-2)-associated protein X (bax) and caspase- 3 were markedly decreased by CMT and HIT, compared with those in the MI group. Expression levels of bcl-2, the classic anti-apoptotic marker, exhibited a significant reduction in the MI group compared with those of the sham group $(\mathrm{P}<0.05)$; and a significant increase in the CMT and HIT groups compared with those of the MI group $(\mathrm{P}<0.05)$. Notably, the expression levels of bax, bcl-2 and caspase- 3 were altered approximately equally in the two exercise groups and no significant differences were detected between the two groups.

CMT and HIT ameliorate oxidative stress of the infracted myocardium. As shown in Fig. 3A, the two forms of AET used in the present study markedly decreased the expression of MDA ( $\mu \mathrm{mol} / \mathrm{mg}$ protein) compared with that of the MI group (CMT: $3.41 \pm 0.13$ vs. $6.53 \pm 0.21, \mathrm{P}<0.05$; HIT: $3.03 \pm 0.17$ vs. $6.53 \pm 0.21, \mathrm{P}<0.05)$. Although CMT and HIT significantly increased the concentration of SOD (U/mg protein), the net effect was minor (CMT: $77.1 \pm 14.9$ vs. $70.2 \pm 15.1, \mathrm{P}<0.05$; HIT: $79.0 \pm 13.2$ vs. $70.2 \pm 15.1, \mathrm{P}<0.05$; Fig. $3 \mathrm{~B})$. By contrast, the increase in GPx (U/mg protein) concentration induced by AET was more marked, accounting for 31.3 and $40.9 \%$ greater increases than that of the MI group $(\mathrm{P}<0.05$; Fig. 3C). Notably, HIT was significantly superior to CMT in elevating the concentration of GPx; although this effect was not observed in the expression of MDA or SOD.

CMT and HIT influence CPT-1, PFK-1 and ATP synthesis of the infarcted myocardium. MI induced a significant downregulation in the mRNA expression and maximal activity of CPT-1 (Fig. 4A and B). AET exerted a small but significant 

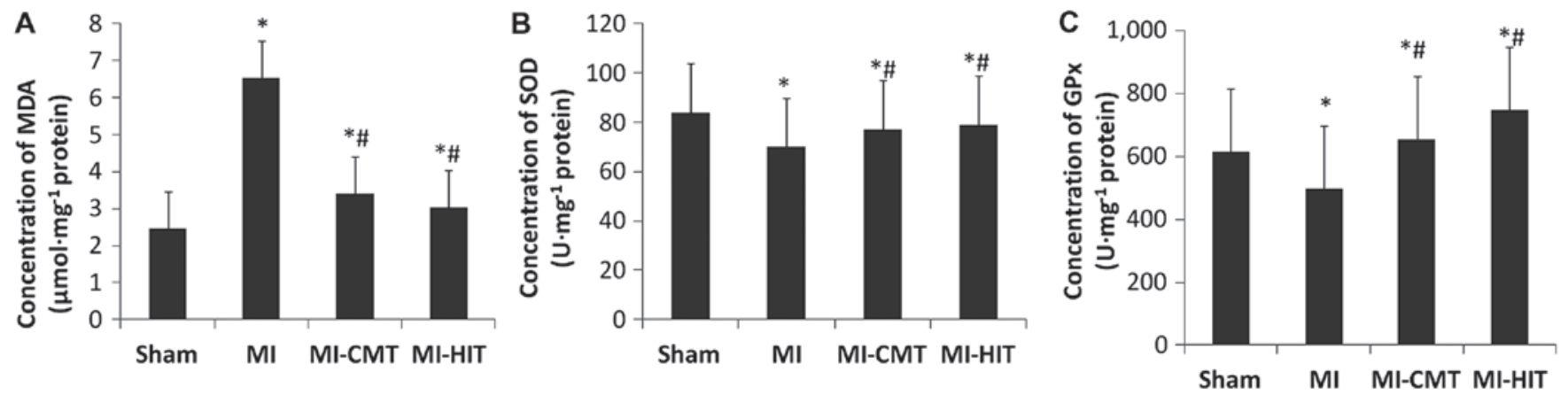

Figure 3. Effects of CMT and HIT on indicators of oxidative stress in the post-MI myocardium. Effect of CMT and HIT on the concentration of (A) MDA ( $\mu \mathrm{mol} / \mathrm{mg}$ protein), (B) SOD (U/mg protein) and (C) GPx (U/mg protein). These indicators of oxidative stress were measured using commercial ELISA kits. Data are expressed as the mean \pm standard deviation. ${ }^{*} \mathrm{P}<0.05$ vs. the sham group; ${ }^{\prime \prime} \mathrm{P}<0.05$ vs. the MI group. MI, myocardial infarction; CMT, continuous moderate training; HIT, high-intensity interval training; MDA, malondialdehyde; SOD, superoxide dismutase; GPx, glutathione peroxidase.
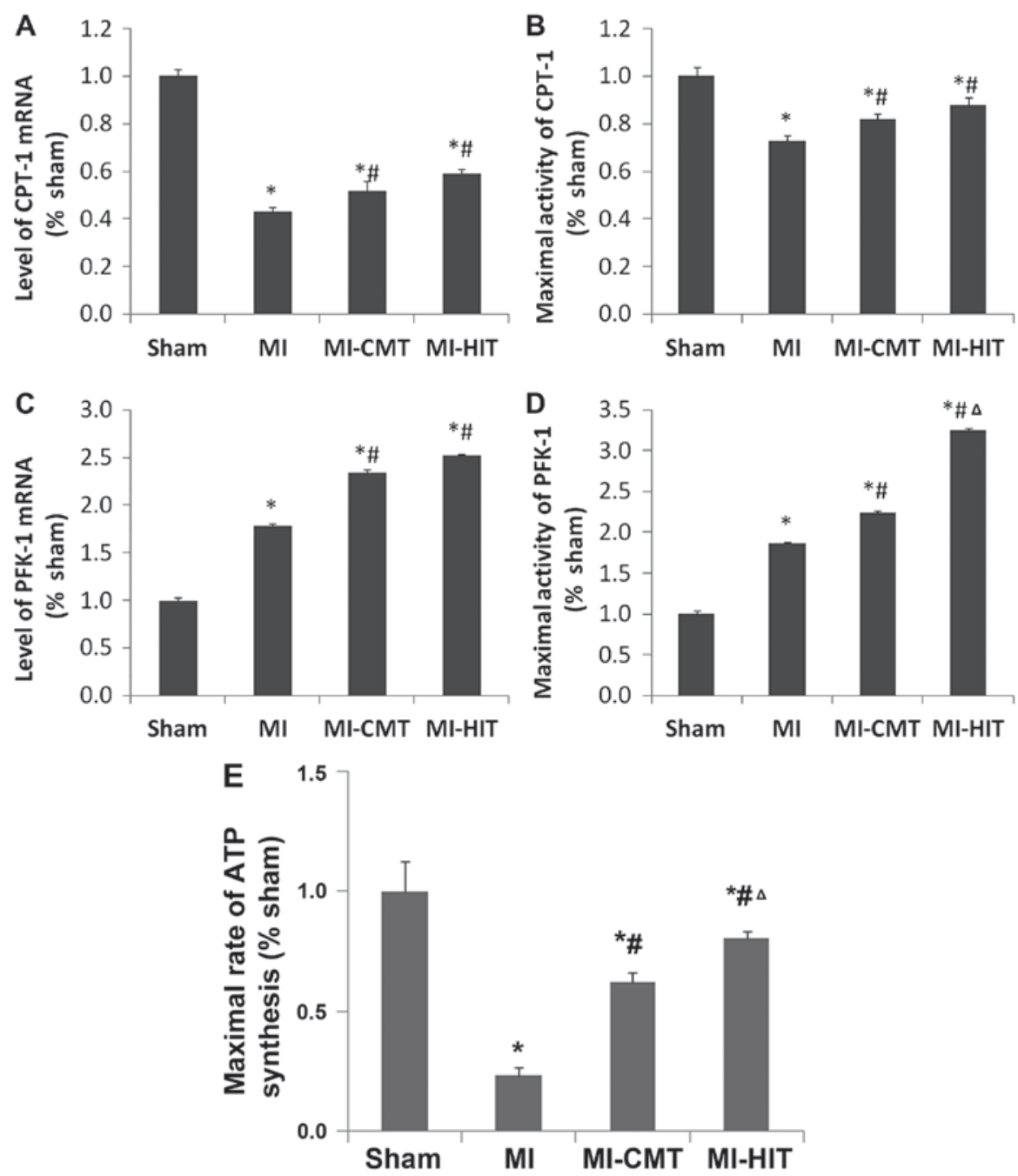

Figure 4. Effects of CMT and HIT on CPT-1, PFK-1 and ATP synthesis in the post-MI myocardium. (A) Effects of CMT and HIT on mRNA expression levels of CPT-1. (B) Effects of CMT and HIT on the maximal activity of CPT-1. (C) Effects of CMT and HIT on mRNA expression levels of PFK-1. (D) Effects of CMT and HIT on the maximal activity of PFK-1. (E) Effects of CMT and HIT on the maximal rate of ATP synthesis. The normalized fold-changes of the CPT-1 and PFK-1 mRNA expression are expressed as $2^{-\triangle \Delta C}$. The mRNA levels and activity of CPT-1 and PKF-1 were normalized to the sham group and are expressed as the mean \pm standard deviation of three independent experiments per group. ${ }^{*} \mathrm{P}<0.05$ vs. the sham group; ${ }^{*} \mathrm{P}<0.05$ vs. the $\mathrm{MI}$ group; ${ }^{\Delta} \mathrm{P}<0.05$ vs. the MI+CMT group. MI, myocardial infarction; CMT, continuous moderate training; HIT, high-intensity interval training; CPT-1, carnitine palmitoyltransferase-1; PFK-1, phosphofructokinase 1; ATP, adenosine triphosphate.

effect on CPT-1 expression, and mRNA expression and maximal activity were enhanced. No significant differences were observed between the two exercise groups. By contrast, the effects of aerobic exercise on mRNA expression and 
A

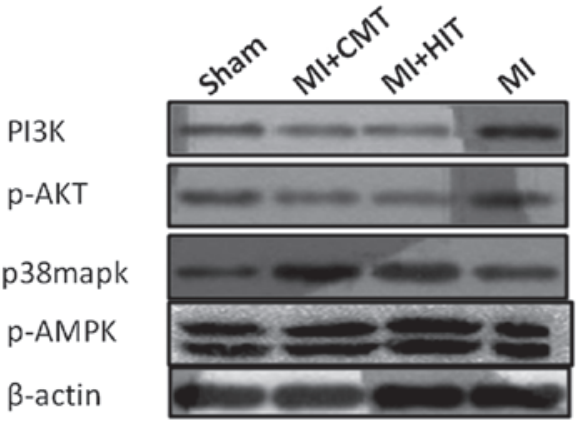

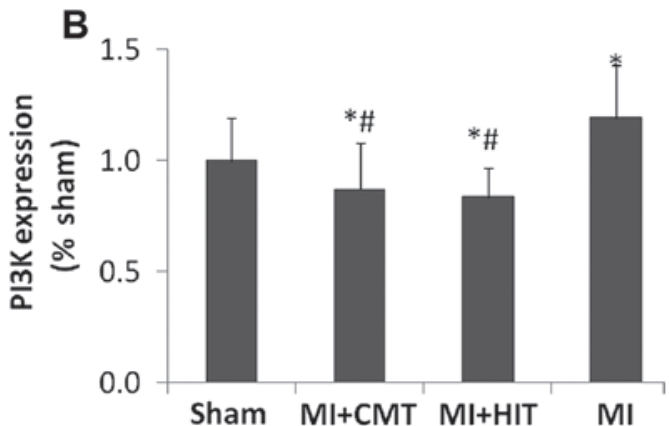

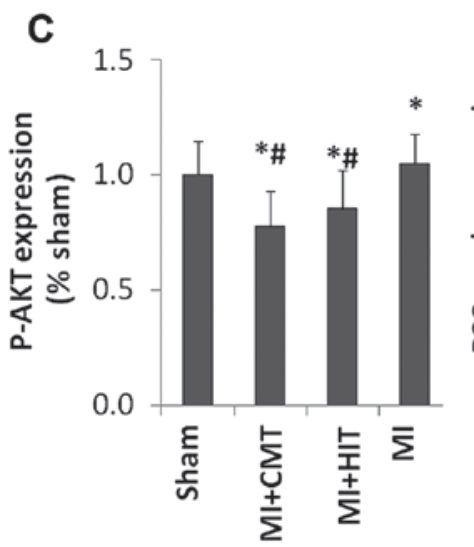

D
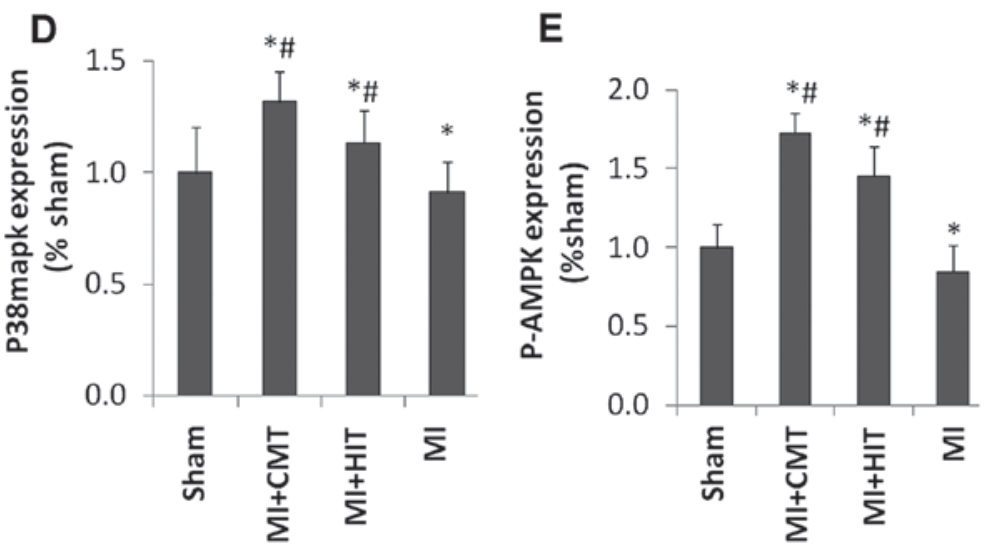

Figure 5. Effects of CMT and HIT on the expression of associated key signaling pathway proteins in the post-MI myocardium. (A) Representative western blot analysis results. Effects of CMT and HIT on protein expression of (B) PI3K, (C) p-AKT, (D) p38mapk and (E) p-AMPK. Protein expression levels were determined by western blotting. The data were normalized to the sham group and were expressed as the mean \pm standard deviation of three independent experiments per group. " $\mathrm{P}<0.05$ vs. the sham group; ${ }^{"} \mathrm{P}<0.05$ vs. the MI group. MI, myocardial infarction; CMT, continuous moderate training; HIT, high-intensity interval training; PI3K, phosphatidylinositol-3-kinase; p-, phosphorylated; mapk, mitogen-activated protein kinase; AMPK, adenosine monophosphate activated protein kinase.

maximal activity of PFK-1 were markedly greater, and the effects of HIT on PFK-1 maximal activity were greater than those of CMT (Fig. 4C and D). HIT induced a greater increase in the maximal activity of PFK-1 than CMT $(3.25 \pm 0.03$ vs. $2.24 \pm 0.02, \mathrm{P}<0.05)$. In addition, aerobic training, and particularly HIT $(\mathrm{P}<0.05)$, significantly increased the maximal rate of ATP synthesis compared with that of the MI group $(0.62 \pm 0.04$ and $0.80 \pm 0.03$ vs. $0.23 \pm 0.03$; P<0.05; Fig. 4E).

CMT and HIT influence the expression of associated signaling pathway proteins. As shown in Fig. 5A-C, the levels of PI3K and p-AKT were significantly enhanced in the MI group, compared with those of the sham group; but CMT and HIT effectively attenuated this effect $(\mathrm{P}<0.05)$. The two forms of aerobic training also abated expression of $\mathrm{p} 38 \mathrm{mapk}$ protein compared with that of the MI group $(\mathrm{P}<0.05 ; \mathrm{P}<0.05$; Fig. 5A and D). In addition, as indicated in Fig. 5A and E, AET, and HIT in particular, effectively enhanced the expression of p-AMPK, which was attenuated in the MI group $(\mathrm{P}<0.05$; $\mathrm{P}<0.05)$.

\section{Discussion}

The modifications exerted by distinct forms of AET on post-MI cardiac functional recovery has previously been rarely reported. In the present study, the effects of two forms of AET, CMT and HIT, on exercise capability and the recovery of cardiac function in a post-MI rat model were compared. In addition, the effects of AET on associated pathophysiological processes and signaling pathways that may contribute to the recovery of cardiac function, including apoptosis, oxidative stress and glucolipid metabolism, were investigated. The results indicated that HIT was superior to CMT with regard to enhancing exercise capability and cardiac functional recovery in post-MI rats. In addition, the results demonstrated that HIT was superior to CMT in terms of alleviating oxidative stress, ameliorating glycolipid metabolism and enhancing ATP production. However, with regard to attenuating apoptosis, no significant differences were observed.

Increasing evidence has demonstrated that CMT may significantly attenuate apoptosis in the rat heart, as indicated by changes in the levels of the apoptosis index, cleaved caspase- 3 levels and caspase- 3 activity $(13,15,29)$. The results of the present study regarding the effect of CMT on apoptosis in the post-MI heart did not contradict these results. However, the effect of HIT on apoptosis of the post-MI myocardium had previously remained to be elucidated. By controlling the total exercise time and distance between the CMT and HIT group, the results of the present study revealed that HIT exerted a similar level of influence on the levels of pro-apoptotic and anti-apoptotic proteins as CMT.

A growing body of evidence has demonstrated that aerobic exercise may alleviate oxidative stress in a post-MI rat model. One previous study demonstrated that only high-intensity 
exercise and moderate-intensity exercise of long duration were able to effectively upregulate SOD activity in the ventricular myocardium, while short durations of moderate-intensity exercise failed to achieve this effect (30). Another study investigating HIT indicated that oxidative stress marker superoxide anion and MDA levels were decreased by AET (31). However, notably, there was significant diversity amongst the training protocols utilized in previous reports and the adaptation of oxidative stress to AET depended upon the type, duration and intensity of exercise (30-34). As such, according to these previous reports, direct comparisons of distinct forms of exercise training on oxidative stress are difficult and to the best of our knowledge has not been done before. In the present study, particular attention was paid to controlling the total training time and distance between the various exercise groups during the training protocol, which was designed to ameliorate potential interference factors to the greatest extent. The results indicated that CMT and HIT were able to attenuate oxidative stress in the post-MI heart, which was consistent with other reports (31). Additionally, it was demonstrated that HIT exerted an equal influence on the levels of MDA and SOD, while it induced a greater change in the levels of GPx, in comparison to that of CMT.

The effects of AET on glucose metabolism of the myocardium following ischemic insult have rarely been reported previously and the results have been contradictory. Broderick et al (35) indicated that exercise training was able to restore decreased myocardial glycolysis in diabetes rats and enhance myocardial glycolysis during ischemia. However, in another study, glycolysis was found to be $25-30 \%$ lower prior to and following ischemia in the heart of exercise-trained rats than that of the control in a rat model of I/R (36). An accompanying improvement in cardiac function was also observed in these two studies. In the present study, glycolysis of cardiomyocytes in the post-MI rat model was significantly enhanced following AET. The models used in these studies possessed varying basic metabolic hypotypes, which may account for the discrepancies observed. The potential mechanisms underlying this effect remain elusive; however, it was hypothesized that this may be associated with the concentration of glucose transporter-4 which may determine the rate of glucose metabolism to a large extent (37) and is potentially increased following AET (38). As to the effect of AET on myocardial fatty acid oxidation, the results of the present study indicated that aerobic exercise significantly increased the mRNA expression and activity of CPT-1, and the expression of p-AMPK. CPT-1 is the rate-limiting enzyme for fatty acid $\beta$-oxidation and is regulated by the AMPK/ACC signaling pathway (39). Burelle et al (36) reported that exercise training increased myocardial palmitate oxidation by $50-65 \%$, compared with that of the sedentary rats prior to and following ischemia. Conversely, another study in a normal heart model found that fatty acid oxidation was not influenced by exercise (40). The results of the present study were consistent with those of Burelle et al (36), and indicated that exercise may exert a differential effect on fatty acid oxidation in the normal and ischemic myocardium. Additionally, the results of the present study revealed that glycolysis was enhanced to a greater extent than fatty acid oxidation, which would increase the proportion of glucose metabolism in total ATP production and alleviate the damage that induced by excessive fatty acid oxidation following ischemic insult in cardiomyocytes.

Improvements in exercise capability following regular and effective exercise training should mainly be attributed to the overall functional enhancement of the motor and cardiopulmonary systems. Moreira et al (10) utilized a similar exercise protocol to that used in the present study and found that skeletal muscle adaptation to HIT and CMT was analogous. Therefore, the results of the present study may in part explain the fact that HIT frequently results in a more favorable increase in exercise capability than CMT for MI patients. In addition, improvements of additional systems and elements may also contribute to the improvement in exercise capacity.

Although HIT has been utilized in the field of medical rehabilitation for decades, the standardized exercise protocol for optimal clinic outcome has remained under debate. The diversity of regimens applied in previous studies also makes it difficult to perform a crosswise comparative analysis among them. Therefore, a series of experiments with standardized protocols performed under strict control are required for the effective determination of the most favorable exercise regimens.

In conclusion, the present study demonstrated that HIT was superior to CMT in improving cardiac function and exercise capability in a post-MI rat model. Furthermore, HIT exerted more favorable effects with regard to alleviating oxidative stress, ameliorating glucolipid metabolism and enhancing ATP production, than those of CMT. However, HIT and CMT were almost equal in their ability to attenuate apoptosis. The current study provided underlying evidence that HIT is more preferable and effective for cardiac rehabilitation as a novel form of aerobic exercise training.

\section{Acknowledgements}

The present study was funded by grants from the 12th Five-year Science and Technology Support Program of the Ministry of Science and Technology of China (grant no. 2013BAI06B02). The authors would like to express their gratitude to Professors Tiemin Ma and Xin Ma from Beijing University of Chinese Medicine (Beijing, China) for their assistance in establishing the rat model.

\section{References}

1. Nagayama M, Itoh $\mathrm{H}$ and Maeda T: Cardiac rehabilitation for patients with acute myocardial infarction. Nihon Rinsho 69 (Suppl 9): S203-S209, 2011 (In Japanese).

2. Achttien RJ, Staal JB, van der Voort S, et al; Practice Recommendations Development Group: Exercise-based cardiac rehabilitation in patients with coronary heart disease: a practice guideline. Neth Heart J 21: 429-438, 2013.

3. Oldridge N: Exercise-based cardiac rehabilitation in patients with coronary heart disease: meta-analysis outcomes revisited. Future Cardiol 8: 729-751, 2012.

4. Moholdt T, Aamot IL, Granøien I, et al: Aerobic interval training increases peak oxygen uptake more than usual care exercise training in myocardial infarction patients: a randomized controlled study. Clin Rehabil 26: 33-44, 2012.

5. Moholdt T, Aamot IL, Granøien I, et al: Long-term follow-up after cardiac rehabilitation: a randomized study of usual care exercise training versus aerobic interval training after myocardial infarction. Int J Cardiol 152: 388-390, 2011.

6. Maiorana A: Interval training confers greater gains than continuous training in people with heart failure. J Physiother 58: 199, 2012. 
7. Kemi OJ and Wisløff U: Mechanisms of exercise-induced improvements in the contractile apparatus of the mammalian myocardium. Acta Physiol (Oxf) 199: 425-439, 2010.

8. Garza MA, Wason EA and Zhang JQ: Cardiac remodeling and physical training post myocardial infarction. World J Cardiol 7: $52-64,2015$

9. Kida K, Osada N, Akashi YJ, Sekizuka H, Omiya K and Miyake F: The exercise training effects of skeletal muscle strength and muscle volume to improve functional capacity in patients with myocardial infarction. Int J Cardiol 129: 180-186, 2008.

10. Moreira JB, Bechara LR, Bozi LH, et al: High-versus moderate-intensity aerobic exercise training effects on skeletal muscle of infarcted rats. J Appl Physiol (1985) 114: 1029-1041, 2013

11. Lee Y and Gustafsson AB: Role of apoptosis in cardiovascular disease. Apoptosis 14: 536-548, 2009.

12. Tullio F, Angotti C, Perrelli MG, Penna C and Pagliaro P: Redox balance and cardioprotection. Basic Res Cardiol 108: 392, 2013.

13. Quindry JC, Hamilton KL, French JP, et al: Exercise-induced HSP-72 elevation and cardioprotection against infarct and apoptosis. J Appl Physiol (1985) 103: 1056-1062, 2007.

14. Chen TI, Shen YJ, Wang IC and Yang KT: Short-term exercise provides left ventricular myocardial protection against intermittent hypoxia-induced apoptosis in rats. Eur J Appl Physiol 111: 1939-1950, 2011.

15. Wambolt RB, Lopaschuk GD, Brownsey RW and Allard MF Dichloroacetate improves postischemic function of hypertrophied rat hearts. J Am Coll Cardiol 36: 1378-1385, 2000.

16. Lopaschuk GD, Spafford MA, Davies NJ and Wall SR: Glucose and palmitate oxidation in isolated working rat hearts reperfused after a period of transient global ischemia. Circ Res 66: 546-553, 1990.

17. Egstrup M, Kistorp CN, Schou M, et al: Abnormal glucose metabolism is associated with reduced left ventricular contractile reserve and exercise intolerance in patients with chronic heart failure. Eur Heart J Cardiovasc Imaging 14: 349-357, 2013.

18. Reichkendler MH, Auerbach P, Rosenkilde M, et al: Exercise training favors increased insulin-stimulated glucose uptake in skeletal muscle in contrast to adipose tissue: a randomized study using FDG PET imaging. Am J Physiol Endocrinol Metab 305 E496-E506, 2013

19. Bourlier V, Saint-Laurent C, Louche K, et al: Enhanced glucose metabolism is preserved in cultured primary myotubes from obese donors in response to exercise training. J Clin Endocrinol Metab 98: 3739-3747, 2013.

20. Castorena CM, Arias EB, Sharma N and Cartee GD: Postexercise improvement in insulin-stimulated glucose uptake occurs concomitant with greater AS160 phosphorylation in muscle from normal and insulin-resistant rats. Diabetes 63: 2297-2308, 2014

21. Franke TF, Hornik CP, Segev L, Shostak GA and Sugimoto C: PI3K/Akt and apoptosis: size matters. Oncogene 22: 8983-8998, 2003.

22. Blanc A, Pandey NR and Srivastava AK: Synchronous activation of ERK 1/2, p38mapk and $\mathrm{PKB} /$ Akt signaling by $\mathrm{H}_{2} \mathrm{O}_{2}$ in vascular smooth muscle cells: Potential involvement in vascular disease (Review). Int J Mol Med 11: 229-234, 2003.

23. Bijland S, Mancini SJ and Salt IP: Role of AMP-activated protein kinase in adipose tissue metabolism and inflammation. Clin Sci (Lond) 124: 491-507, 2013.
24. Pfeffer MA, Pfeffer JM, Fishbein MC, et al: Myocardial infarct size and ventricular function in rats. Circ Res 44: 503-512, 1979.

25. Høydal MA, Wisløff U, Kemi OJ and Ellingsen O: Running speed and maximal oxygen uptake in rats and mice: practical implications for exercise training. Eur J Cardiovasc Prev Rehabil 14: 753-760, 2007.

26. Kraljevic J, Marinovic J, Pravdic D, et al: Aerobic interval training attenuates remodelling and mitochondrial dysfunction in the post-infarction failing rat heart. Cardiovasc Res 99: 55-64, 2013.

27. Pauly DF and McMillin JB: Importance of acyl-CoA availability in interpretation of carnitine palmitoyltransferase I kinetics. J Biol Chem 263: 18160-18167, 1988.

28. Dumas JF, Goupille C, Julienne CM, et al: Efficiency of oxidative phosphorylation in liver mitochondria is decreased in a rat model of peritoneal carcinosis. J Hepatol 54: 320-327, 2011.

29. Zhang KR, Liu HT, Zhang HF, et al: Long-term aerobic exercise protects the heart against ischemia/reperfusion injury via PI3 kinase-dependent and Akt-mediated mechanism. Apoptosis 12: 1579-1588, 2007.

30. Powers SK, Criswell D, Lawler J, et al: Rigorous exercise training increases superoxide dismutase activity in ventricular myocardium. Am J Physiol 265 (6 Pt 2): H2094-H2098, 1993.

31. Jiang HK, Miao Y, Wang YH, et al: Aerobic interval training protects against myocardial infarction-induced oxidative injury by enhancing antioxidase system and mitochondrial biosynthesis. Clin Exp Pharmacol Physiol 41: 192-201, 2014.

32. Barbosa VA, Luciano TF, Vitto MF, et al: Exercise training plays cardioprotection through the oxidative stress reduction in obese rats submitted to myocardial infarction. Int J Cardiol 157: 422-424, 2012

33. Pinho CA, Tromm CB, Tavares AM, et al: Effects of different physical training protocols on ventricular oxidative stress parameters in infarction-induced rats. Life Sci 90: 553-559, 2012.

34. Frederico MJ, Justo SL, Da Luz G, et al: Exercise training provides cardioprotection via a reduction in reactive oxygen species in rats submitted to myocardial infarction induced by isoproterenol. Free Radic Res 43: 957-964, 2009.

35. Broderick TL, Poirier P and Gillis M: Exercise training restores abnormal myocardial glucose utilization and cardiac function in diabetes. Diabetes Metab Res Rev 21: 44-50, 2005.

36. Burelle Y, Wambolt RB, Grist M, et al: Regular exercise is associated with a protective metabolic phenotype in the rat heart. Am J Physiol Heart Circ Physiol 287: H1055-H1063, 2004.

37. Garvey WT, Hardin D, Juhaszova M and Dominguez JH: Effects of diabetes on myocardial glucose transport system in rats: implications for diabetic cardiomyopathy. Am J Physiol 264 (3 Pt 2): H837-H844, 1993.

38. Richter EA and Hargreaves M: Exercise, GLUT4 and skeletal muscle glucose uptake. Physiol Rev 93: 993-1017, 2013.

39. Hardie DG and Pan DA: Regulation of fatty acid synthesis and oxidation by the AMP-activated protein kinase. Biochem Soc Trans 30: 1064-1070, 2002.

40. Zonderland ML, Bär PR, Reijneveld JC, Spruijt BM, Keizer HA and Glatz JF: Different metabolic adaptation of heart and skeletal muscles to moderate-intensity treadmill training in the rat. Eur J Appl Physiol Occup Physiol 79: 391-396, 1999. 